\title{
Dental stem cells as a potential tools for regeneration of tooth structures
}

\author{
AGNIESZKA MIELCZAREK ${ }^{1}$, MAEGORZATA KLUKOWSKA², TOMASZ GEDRANGE \\ SYLWIA MAEGORZATA SEOTWIŃSKA ${ }^{I}$
}

${ }^{1}$ Department of Conservative Dentistry, Medical University of Warsaw, Poland

2Procter \& Gamble Company, Mason, OH, USA

${ }^{3}$ TU Dresden, Germany

\begin{abstract}
Voluminous research has focused on the identification of sources of adult stem cells in human body. Recent progress on the isolation and regulation of stem cells may enable the future development of novel therapeutic strategies based on the concept of regeneration of damaged or diseased organs. Recently various subpopulations of mesenchymal stem cells were identified in dental tissues, including dental pulp, apical papilla and periodontal ligament. They are considered to offer potential for regeneration of tooth structure and bone. Additionally, dental stem cells can possibly be applied in therapy of other disorders such as Alzheimer's disease, Parkinson's disease or cardiac ischemia. This review describes the stem cell subpopulations derived from dental tissues and their potential role in tooth regeneration and other therapies.
\end{abstract}

Key words: dental stem cells, tooth regeneration.

(Centr Eur J Immunol 2013; 38 (1): 107-110)

A longstanding goal in dental medicine is to maintain tooth and periodontal structures for the lifetime of our patients. Today we attempt to accomplish this first, through application of hygiene for the prevention of both hard and soft tissue diseases. When prevention fails, we aid patients in restoration of their dental function with various treatment procedures. Some of the most advanced treatment procedures today include sophisticated dental restoratives for restoration of tooth tissue, the provision of implants for teeth loss and regenerative materials for reconstruction of alveolar bone. Despite significant progress, biomimetic restored teeth and periodontal structures are invariably deficient versus their natural counterparts. Dentists have long desired the ability to replace damaged dental structures with natural functioning teeth and gums. Today this goal may be within reach due to progress in the field of regenerative medicine and application of stem cell therapies.

The concept of regenerative medicine was introduced to medical nomenclature in the end of last century. Regenerative medicine can be defined as the "process of replacing or regenerating human cells, tissues or organs to restore or establish normal function" [1]. This field holds the promise of novel therapy by regeneration damaged tissues and organs and/or stimulation of the body's own repair mechanisms. Regenerative therapy may be accomplished by variety of approaches. Among regenerative techniques the most advanced include: 1) cell therapy, which is based on application of cell suspensions for the stimulation of therapeutic effects, and 2) tissue engineering. For the latter, the restoration of parts of organs or whole organs is accomplished by application of stem cells. This approach is in fact the most promising for dental applications. As background, stem cells are biological cells found in all multicellular organisms, that can divide (through mitosis) and differentiate into diverse specialized cell types [2]. Generally two kinds of stem cells are recognized in human body: embryonic stem cells (ESC) and somatic or adult stem cells (ASC). Human embryonic stem cells are pluripotent (can differentiate into cells coming from 3 germ layers) and are derived from four to five day old embryos in blastocyste stage. In embryonic stem cells two properties are particularly important: the capacity to differentiate into any cell

Correspondence: Agnieszka Mielczarek, Department of Conservative Dentistry, Medical University of Warsaw, Miodowa 18, 00-246 Warszawa, Poland, e-mail: agam@amwaw.edu.pl 
type in the body and the ability to self-replicate for numerous generations [3]. There are still many ethical and moral controversies over the use of embryonic stem cells, mainly concerned with the requiring of the destruction of human embryos early in development. Additionally, from an applied technical perspective, unlimited proliferation and differentiation capacity is recognized as a great disadvantage of human ESC application. The possibility of uncontrolled cell proliferation may lead to embryonic tumor formation $[4,5]$. Due to these dilemmas little attempt has been made towards the use of ESC in dentistry and much more attention has been focused on ASC applications.

ASC's are slow-cycling, undifferentiated cells, surrounded by neighboring cells and extracellular matrix [6]. The interaction of cells and environment create the special niches for the existence of such adult stem cells. Their proliferation can be initiated by disease or tissue injury [7]. For a long time, adult stem cells from bone marrow were widely used in regenerative medicine procedures. Two types of marrow stem cells were identified of potential use: hematopoietic stem cells, which are the precursors of all types of blood cells and stromal stem cells [8]. A specific form of the stromal stem cells are marrow-derived mesenchymal stem cells (MSC). Besides bone marrow, somatic stem cells have been identified in a variety of tissues, such as skin, nerve, muscle, liver, pancreas, retina, synovium or cartilage [9]. The difficulty of cell collection from marrow and trauma associated with surgical procedure prompted scientists to search for alternative sources of postnatal stem cells.

The cells which are involved in odontogenesis process have ectodermal or mezenchymal origins. Ectodermal cells are responsible for enamel formation and they are lost after tooth eruption. Therefore, only MSCs cells are available for cell therapy. Yalvac et al. demonstrated that human tooth germs contain multipotent cells that give rise to dental and periodontal structures [10]. Since then, it has been discovered that stem cells derived from dental tissues seem to be particularly attractive for future applications because their combined properties.

A number of postnatal, dental-origin stem cells have now been identified according to localization in tooth structures. These include:

- dental pulp stem cells (DPSC),

- dental follicle stem cells (DFSC),

- human exfoliated deciduous teeth stem cells (SHED),

- apical papilla stem cells (SCAP),

- periodontal ligament stem cells (PDLSC).

Dental tissue stem cells have been obtained from impacted teeth. The development of wisdom teeth starts postnatally, and their completed root calcification takes several years what makes them a great potential source of DPSC, PDL and SCAP cells [11].

The potential of stem cells derived from dental pulp for use in tissue regeneration was first recognized based on the evidence of reparative dentine formation after pulp capping resulting from the pulp exposure to advanced caries processes or after injury episodes. Gronthos et al. 2000 were the first one to report the isolation of stem cells from the dental pulp of wisdom teeth [12]. Subsequently, Miura et al. obtained ectomesenchymal stem cells from exfoliated deciduous teeth [13]. Some in vitro and in vivo study have been undertaken to determine the regenerative potential of DPSCs. The study results showed that DPSCs can differentiate to odontoblasts, osteoblasts, endothelocytes, smooth muscle cells, adipocytes, chondrocytes, and neurons [14]. The application of odontotropic medications was shown to induce pulpal progenitor cells to differentiate into odontoblasts. Efforts have also been made to use DPSCs in perforated furcations treatment [15].

Dental follicle stem cells (DFSCs) are isolated from mesenchymal tissue localized around developing tooth germ. This source of stem cells can be easily obtained from follicles of impacted third molars [11]. DFSCs are recognized as progenitors for cementoblasts, PDL stem cells, osteoblasts as well as neural cells. DFSCs have the capacity to induce calcification processes in vitro and in vivo. Experiments undertaken with DFSCs revealed their potential for use in tissue engineering applications, including periodontal and bone regeneration DFSCs are recognized as osteogenesis and dentinogenesis inductors, but have not shown ability to produce dentin-pulp complex formation $[7,16]$.

SHED cells can be easily accessible from coronal pulp of exfoliated deciduous teeth. These cells have the capacity to: proliferate with high rate, induce bone formation, create dentine, increased cell-population doublings, induce recipient cell-mediated bone formation in vivo, sphere-like cell-cluster formation and differentiate to other non-mesenchymal dental cells. SHED expressed neuronal and glial cell markers, which may be related to the neural crest-cell origin of the dental pulp. Neural crest cells play a pivotal role in embryonic development, giving rise to a variety of cell types such as neural cells, pigment cells, smooth muscle, craniofacial cartilage, and bone [13]. Experiments examining replacement of infected dental pulp with SHED stem cells revealed the possibility of pulp-like tissue formation, with cellular and structural similarities to original dental pulp. Noteworthy is also the possibility of clinical application of SHED cells in Parkinson's disease therapy [17]. As a practical benefit, SHED are capable of providing adequate numbers of cells for potential clinical application.

A unique population of dental stem cells (SCAP) has been identified in the apical papilla-structure present temporarily in the apex of developing tooth root. SCAP cells can differentiated into odontoblasts and have embryoniclike characteristics. SCAP appear to be the main source of primary odontoblasts, responsible for the formation of root dentin. Root apical papilla stem cells exhibit high rates of 
proliferation and in combination with PDL stem cells have the potential to create a biological tooth root, as an alternative for dental implants. Huang et al. examined the potential role of SCAP in continued root formation [18]. Human immature incisor was injured with pulp exposure. During the endodontic treatment the pulp was extirpated, while the apical papilla was retained. After root canal treatment continued root-tip formation was observed. The ability of SCAP cells to promote root formation was confirmed in the other study [19]. The bio-root obtained in this study was covered by periodontal ligament tissue and appeared to have a natural relationship with the surrounding bone. Unfortunately, the formed root did not exhibit sufficient mechanical strength. This is a focus of continued investigations.

Another source of stem cells for potential use in regenerative therapy is the peridontium. Periodontal ligament contain a population of progenitor cells (PDLSC), which can differentiate to produce cementoblasts-like cells, adipocytes and connective tissue rich in collagen I [20, 21]. The identification of these kinds of cells creates new possibilities in periodontal tissue regeneration field. Gault et $a l$. in an animal study, investigated the possibility of using PDL cells to create periodontal ligament structure around dental implants. PDL cells were isolated and cultured in a bioreactor on titanium pins. After the formation of multiple cellular layers, pins were implanted in dental alveoli. As a result of the experiment cell-covered implants integration were observed without adverse effects, and induced bone formation in their vicinity [22]. Additional experiments examined the potential use of allogeneic periodontal ligament stem cells (PDLSCs) in therapy of experimentally induced periodontitis in a miniature pig. The authors achieved a significant periodontal tissue regeneration in both the autologous and the allogeneic transplantation groups. The study demonstrated that PDL stem cells can stimulate repairing processes in allogeneic bone defects. There was no evidence of immunological rejections in animals receiving the transplanted allogeneic PDLSCs [23].

Of course in order to be successfully used for tissue regeneration dental stem cells have to be isolated, identified and cultured under defined conditions. The specific markers essential in the identification, characterization and isolation of dental derived stem cells have now been carefully examined. STRO-1, a trypsin-resistant cell-surface antigen and Stro-4, which binds to heat shock protein $90 \beta$, are recognized as a basic, commonly used cell markers responsible for dental mesenchymal stem cells identification. Another marker used for characterization and differentiation of stem cells includes the osteoblast marker osteocalcin, which is also used as a stem cell marker of DPSCs. Embryonic stem cell markers Oct-4, Nanog, SSEA-3, SSEA-4, TRA-1-60 and TRA-1 have also affinity to immature dental pulp stem cells [24].

Stem cells have been identified and differentiated in various tissue types of the body. Here we have described how dental structures harbor several niches of multipotent stem cells. While significant progress has been made with these dental stem cells, we have not yet been able to directly utilize dental stem cells in human tooth regeneration. Improved isolation techniques and refinements in growth control system must be established and tested to achieve this goal. Contemporary researchers are currently focused on manipulation of intrinsic or extrinsic signaling molecules for host stem cells activation and mobilization in dental applications. With improved understanding, research studies provide hope of near future application of stem cell based tooth and periodontal support structure regeneration for patients.

The authors thank Dr. Donald White for assistance in manuscript preparation.

\section{References}

1. Mason C, Dunnill P (2008): A brief definition of regenerative medicine. Regen Med 3: 1-5.

2. Mao JJ (2008): Stem cells and the future of dental care. N Y State Dent J 74: 20-24.

3. Honda MJ, Fong H, Iwatsuki S, et al. (2008): Tooth-forming potential in embryonic and postnatal tooth bud cells. Med Mol Morphol 4: 183-192.

4. Harada H, Mitsuyasu T, Toyono T, et al. (2002): Epithelial stem cells in teeth. Odontol 90: 1-6.

5. Ryu JK, Kim J, Cho SJ, et al. (2004): Proactive transplantation of human neural stem cells prevents degeneration of striatal neurons in a rat model of Huntington disease. Neurobiol Dis 16: 68-77.

6. Bluteau G, Luder HU, De Bari C, Mitsiadis TA (2008): Stem cells for tooth engineering. Eur Cell Mater 16: 1-9.

7. Sandhu SS, Nair M (2009): Stem cells: Potential implications for tooth regeneration and tissue engineering in dental science. People J Scien Res 2: 41-45.

8. Avasthi S, Srivastava RN, Singh A, et al. (2008): Stem cell: past, presentand future- a review article. Inter J Med Up 3: 22-30.

9. Pounto I, Giannoudis PV (2005): Biology of mesenchymal stem cells. Injury Int J Care Injured 36: 8-12.

10. Yalvac ME, Ramazanoglu M, Rizvanov AA, et al. (2010): Isolation and characterization of stem cells derived from human third molar tooth germs of young adults: implications in neovascularization, osteo-, adipo- and neurogenesis. Pharmacogenomics J 10: 105-113.

11. Volponi AA, Pang Y, Sharpe PT (2010): Stem cell-based biological tooth repair and regeneration. Trends Cell Biol 20: 715722.

12. Gronthos S, Mankani M, Brahim J, et al. (2000): Postnatal human dental pulp stem cells (DPSCs) in vitro and in vivo. Proc Natl Acad Sci U S A 97: 13625-13630.

13. Miura M, Gronthos S, Zhao M, et al. (2003): SHED: stem cells from human exfoliated deciduous teeth. Proc Natl Acad Sci U S A 100: 5807-5812.

14. Estrela C, Alencar AH, Kitten GT, et al. (2011): Mesenchymal stem cells in the dental tissues: perspectives for tissue regeneration. Braz Dent J 22: 91-98.

15. Prescott RS, Alsanea R, Fayad MI, et al. (2008): In vivo generation of dental pulp-like tissue by using dental pulp stem 
cells, a collagen scaffold, and dentin matrix protein 1 after subcutaneous transplantation in mice. J Endod 34: 421-426.

16. Honda MJ, Imaizumi M, Tsuchiya S, Morsczeck C (2010): Dental follicle stem cells and tissue engineering. J Oral Sci 52: 541-552.

17. Wang J, Wang X, Sun Z, et al. (2010): Stem cells from human exfoliated deciduous teeth can differentiate into dopaminergic neuron-like cells. Stem Cells Dev 19: 1375-1383.

18. Huang GT, Sonoyama W, Chen J, Park SH (2006): In vitro characterization of human dental pulp cells: various isolation methods and culturing environments. Cell Tissue Res 324: 225-236.

19. Sonoyama W, Liu Y, Yamaza T, et al. (2008): Characterization of the apical papilla and its residing stem cells from human immature permanent teeth: a pilot study. J Endod 34: 166-171.

20. Seo BM, Miura M, Gronthos S, et al. (2004): Investigation of multipotent postnatal stem cells from human periodontal ligament. Lancet 364: 149-155.

21. Sonoyama W, Liu Y, Fang D, et al. (2006): Mesenchymal stem cell-mediated functional tooth regeneration in swine. PLoS One 1: e79.

22. Gault P, Black A, Romette JL, et al. (2010): Tissue-engineered ligament: implant constructs for tooth replacement. J Clin Periodontol 37: 750-758.

23. Ding G, Liu Y, Wang W, et al. (2010): Allogeneic periodontal ligament stem cell therapy for periodontitis in swine. Stem Cells 28: 1829-1838.

24. Ulmer FL, Winkel A, Kohorst P, et al. (2010): Stem cells prospects in dentistry. Schweiz Monatsschr Zahnmed 120: 860-872. 